\title{
Nanoscale
}

Check for updates

Cite this: Nanoscale, 2019, 11, 19713

Received 22nd May 2019,

Accepted 2nd September 2019

DOI: $10.1039 / c 9 n r 04375 f$

rsc.li/nanoscale

\section{Strength of bacterial adhesion on nanostructured surfaces quantified by substrate morphometry $\dagger$}

\author{
Christian Spengler, (iD a Friederike Nolle, ${ }^{a}$ Johannes Mischo, ${ }^{a}$ Thomas Faidt, (iD ${ }^{a}$ \\ Samuel Grandthyll, ${ }^{a}$ Nicolas Thewes, ${ }^{a}$ Marcus Koch, ${ }^{b}$ Frank Müller, (D) ${ }^{a}$ \\ Markus Bischoff, (D) ${ }^{c}$ Michael Andreas Klatt (D) $t^{\mathrm{d}}$ and Karin Jacobs (D) *a
}

\begin{abstract}
Microbial adhesion and the subsequent formation of resilient biofilms at surfaces are decisively influenced by substrate properties, such as the topography. To date, studies that quantitatively link surface topography and bacterial adhesion are scarce, as both are not straightforward to quantify. To fill this gap, surface morphometry combined with single-cell force spectroscopy was performed on surfaces with irregular topographies on the nano-scale. As surfaces, hydrophobized silicon wafers were used that were etched to exhibit surface structures in the same size range as the bacterial cell wall molecules. The surface structures were characterized by a detailed morphometric analysis based on Minkowski functionals revealing both qualitatively similar features and quantitatively different extensions. We find that as the size of the nanostructures increases, the adhesion forces decrease in a way that can be quantified by the area of the surface that is available for the tethering of cell wall molecules. In addition, we observe a bactericidal effect, which is more pronounced on substrates with taller structures but does not influence adhesion. Our results can be used for a targeted development of 3D-structured materials for/against bio-adhesion. Moreover, the morphometric analysis can serve as a future gold standard for characterizing a broad spectrum of material structures.
\end{abstract}

\section{Introduction}

Bacterial biofilms can cause serious problems in many medical, biological and industrial applications. ${ }^{1-3}$ Once formed, they are chemically and mechanically robust and therefore very difficult to remove. ${ }^{4,5}$ A promising approach in biofilm prevention is to inhibit the first step of its formation, which is the adhesion of bacterial cells to surfaces. An important substrate property for the adhesion of bacterial cells is the surface topography, which has therefore been addressed in numerous studies, ${ }^{6-9}$ especially in the prevention of biofouling, a subject of ongoing discussion. ${ }^{8-10}$ However, in the literature no universal approach to quantify bacterial adhesion on nanostructured surfaces can be found since previous studies mostly feature two main drawbacks: lack of (i) quantitative adhesion force determination and (ii) detailed surface characterization.

(i) Bacterial adhesion on structured surfaces has often been examined in adsorption experiments by immersing the structured surfaces in bacterial suspensions. ${ }^{11-15}$ Although this approach is intuitive and mimics the natural situation of bacterial colonization quite accurately, the results from different labs are hard to compare since too many parameters are involved that cannot be controlled accurately. For example, the processes of obtaining the number of adhering cells may differ: in some cases, the sample is first dried and then coated with gold in order to count the bacteria later in electron microscopy images ${ }^{11,12}$ or by conductance microbiology. ${ }^{13}$ It is also difficult to describe the rinsing of loosely bound cells and the removal of adsorbed cells for plating or counting in all parameters. These problems can be overcome by quantitative measurements of actual bacterial adhesion forces (the force required to detach the cell from the surface) under controlled conditions. Hence, we used single-cell force spectroscopy (SCFS), a well-established method for quantitative adhesion force measurements of living bacterial cells. ${ }^{16-26}$

(ii) Many studies have used different types of topographically (regularly ${ }^{27-32}$ or randomly ${ }^{11-15,33-36}$ ) structured surfaces 
without a detailed morphological characterization. There are a number of parameters to describe surface morphologies: $:^{37,38}$ for example, the average roughness or the root mean square (RMS) roughness are very descriptive parameters that are, therefore, often used in bacterial adhesion studies. Other measures, such as the skewness or the kurtosis of the surface, are less intuitive, but give additional information about the surface geometry. ${ }^{35,36}$ However, all these parameters are local shape descriptors that are insensitive to global features because they do not distinguish between arbitrary permutations of the positions of different heights. Therefore, these parameters do not fully characterize the surface morphologies, which makes it difficult to compare the results of different studies. One way to overcome these problems in describing the topography are the so-called Minkowski functionals that we therefore used in our study. ${ }^{39}$

Minkowski functionals are comprehensive and efficient shape descriptors from integral geometry ${ }^{40}$ that contain, put simply, the complete additive - and hence robust - shape information (according to Hadwiger's theorem). ${ }^{41}$ Since they are versatile geometric measures, they have already been widely used in statistical physics and pattern analysis (see ref. 42-48 and references therein). There, an integral geometrybased analysis using the Minkowski functionals has been termed morphometry. ${ }^{48}$ With this method, height data of nanostructured surfaces can be analysed using three-dimensional Minkowski functionals or the level sets at different heights using two-dimensional Minkowski functionals (for explanatory figures, see the ESI†).

In 2D, the Minkowski functionals of a domain can be intuitively interpreted as its area, perimeter, and Euler characteristic. The latter is a topological constant, which is given for a compact body by its number of components minus its number of holes.

In this study, we used Staphylococcus aureus (S. aureus), a Gram-positive, biofilm-forming bacterium that is a frequent cause of severe infections, ${ }^{49}$ and measured the adhesion forces of single cells to nanostructured surfaces (see Fig. 1 for an about-to-scale sketch) that are characterized by their Minkowski functionals and show how the strength of bacterial

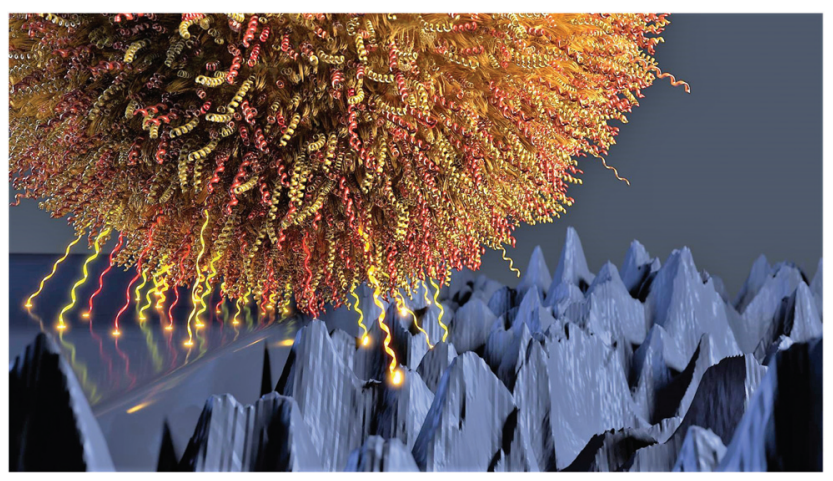

Fig. 1 Bacterial cell adhering to a partially smooth and nanostructured surface (represented by real AFM data). adhesion can be quantified with the help of these functionals. We focus on nanostructures since the radius of the contact area of bacteria like $S$. aureus to flat surfaces is only in the range of some hundred nanometers. ${ }^{50}$

\section{Results and discussion}

\section{Surface morphometry}

In order to create suitable substrates for the quantification of the influence of surface nanostructures on bacterial adhesion, we etched clean silicon wafers in a mixture of fluoric acid, hydrogen peroxide and water for different periods of time (90 s, $180 \mathrm{~s}, 360 \mathrm{~s}) .{ }^{51}$ Beforehand, the surfaces were covered with small gold clusters as catalysts for the etching process. These clusters were applied by physical vapor deposition ${ }^{52}$ and afterwards removed by immersing the wafers in aqua regia. Then, the wafers were rendered hydrophobic because strong bacterial adhesion is governed by hydrophobic interaction. ${ }^{53,54}$ This was achieved by covering them with a self-assembling monolayer of silanes according to a standard recipe ${ }^{55}$ (the quality of the silane monolayer was checked by contact angle measurements and AFM experiments in quantitative nanomechanical mapping; for details, see the ESI†). As shown in Fig. 2a, on the formerly smooth surfaces, etching created characteristic nanostructures, whose lateral and vertical dimensions increased with increasing etching time. The structures feature sharp edges on all surfaces and their shape seems similar in general.

To characterize the surface structures in a quantitative way, the hydrophobized substrates were imaged by atomic force microscopy (AFM) at different positions with high aspect ratio tips. Before further analysis, the surface was reconstructed by unfolding the recorded image and the tip geometry. As a simple parameter - and for comparison to other studies - the RMS roughness of each surface was determined (and was used as label for the different surfaces). Its value increases with increasing etching time: $7 \pm 1 \mathrm{~nm}$ for the $90 \mathrm{~s}$ etched surface, $24 \pm 1 \mathrm{~nm}$ for the $180 \mathrm{~s}$ etched surface, and $35 \pm 1 \mathrm{~nm}$ for the $360 \mathrm{~s}$ etched surface.

For a more detailed characterization, the Minkowski functionals of all AFM images were calculated and averaged over different positions on each surface ${ }^{47}$ (for a visual explanation of the Minkowski functionals of level sets, see Fig. S2 in the ESI $\dagger$ ). ${ }^{45,46}$ Since the maximum height range of the samples varies, the functionals were normalized to their respective maximum height value. In Fig. $2 \mathrm{~b}$ and $\mathrm{c}$, the specific perimeter and the Euler characteristic density are plotted in dependence of this normalized threshold height (absolute values and data of surface area are given in Fig. S3 in the ESI $\dagger$ ). For all surfaces, both functionals have similar shapes, with only their extents varying: the specific perimeter features a very smooth shape with a single maximum for all substrates. The position of the maximum differs slightly between the different substrates (for an explanation, see Fig. S6 in the ESI†). The Euler characteristic density has, in every case, for low threshold heights, a minimum with negative values and at larger heights, a 
a

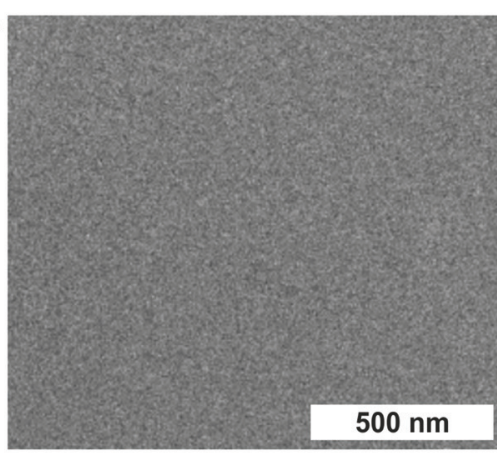

RMS $=0.1 \mathrm{~nm}$

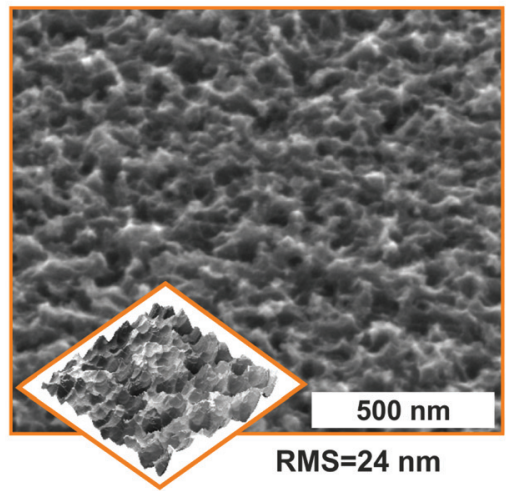

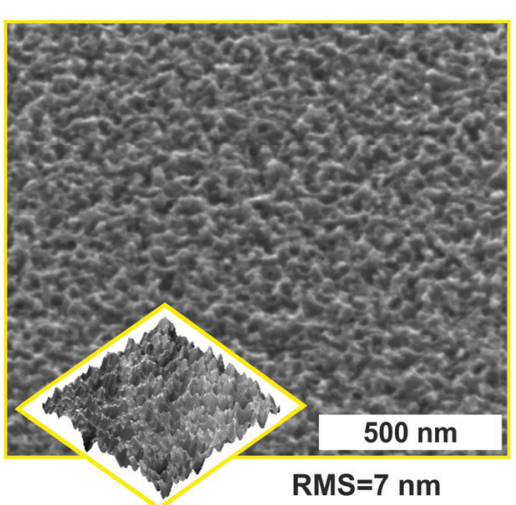

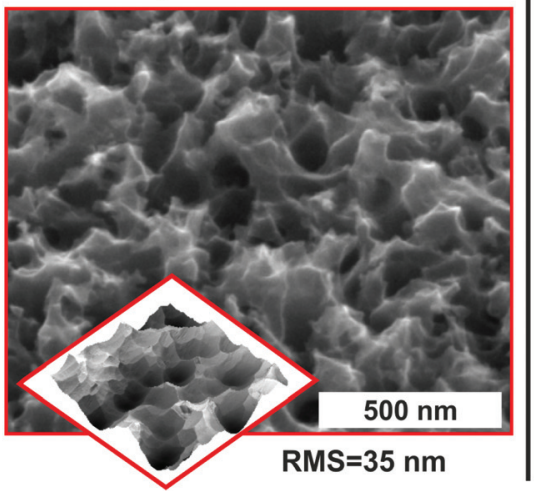

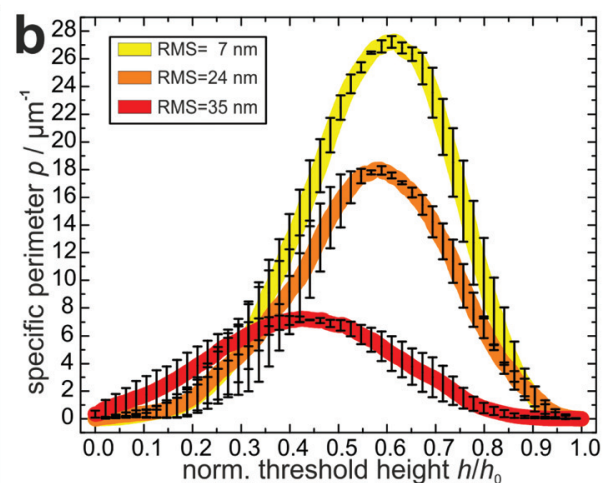

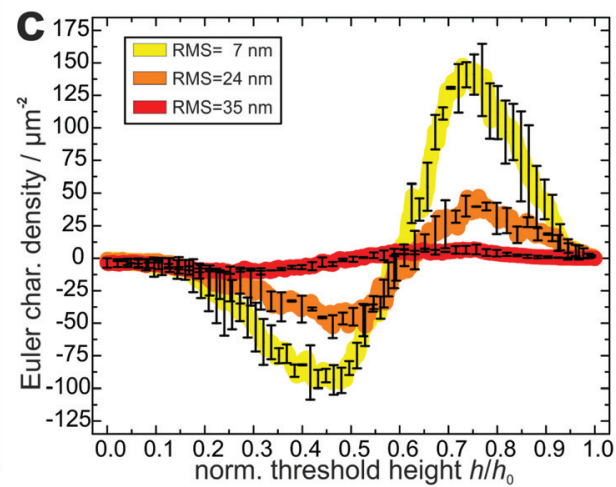

Fig. 2 (a) Scanning electron microscopy (SEM, big) and atomic force microscopy (AFM, small) images of the surfaces before etching and after etching for $90 \mathrm{~s}, 180 \mathrm{~s}$ and $360 \mathrm{~s}$ (from top left to bottom right). SEM images are tilted by $53^{\circ}$. The AFM images show a scan area of $1 \times 1 \mu \mathrm{m}^{2}$ and total $z$-scales of $55 \mathrm{~nm}, 130 \mathrm{~nm}$ and $180 \mathrm{~nm}$, respectively. The RMS values have been determined by AFM. (b and c) Averaged specific perimeter (b) and Euler characteristic density (c) of the nanostructured substrates as a function of the normalized threshold height (for clarity, only for every 20 th data point is an error bar shown).

maximum with positive values. Due to the different RMS values, the peaks and dips, especially for the surface with an RMS value of $35 \mathrm{~nm}$ occur at different values of normalized threshold height. The Minkowski functionals thus characterize quantitative differences between the surfaces. These differences pertain mainly to the absolute values of the specific perimeter and Euler characteristic density: both quantities have the highest values for the surface with $7 \mathrm{~nm}$ RMS roughness, lower values for the surface with $24 \mathrm{~nm}$ RMS roughness and the lowest values for the surface with $35 \mathrm{~nm}$ RMS roughness. This means that the lateral dimensions of the etched structures are smaller for the surfaces that were etched for shorter times.

Overall, the Minkowski analysis confirms the morphological similarity between the surfaces, allowing for a systematic investigation of the influence of differently sized nanostructures on bacterial adhesion. Whether the differences are due to statistical fluctuations, pixelization errors, or physically relevant qualitative differences between the surfaces, is beyond the scope of this study and not relevant for our further analysis.

Moreover, these analyses show that all etched surfaces feature a sub-micron topography with dimensions in the same range as the radius of the bacterial contact area, which is about $150-300 \mathrm{~nm} .{ }^{50}$
Bacterial adhesion is known to be affected not only by surface chemistry, but also by the subsurface of a material through long-ranging van der Waals forces. ${ }^{57}$ Hence, to exclude potential influences originating from differences in surface and subsurface composition, XPS measurements of all substrates were performed before silanization, showing that the roughened surfaces oxidize immediately after etching. Since this surface oxide layer has the same thickness as the oxide layers of the unetched substrates (XPS spectra are given in Fig. S1 in the ESI $\dagger$ ), all surfaces used can be considered chemically identical.

\section{Effect of the nanostructure on bacterial adhesion}

To quantify the impact of the nano-topography on the adhesive strength of bacteria, we performed single-cell force spectroscopy measurements on each etched surface and on a smooth surface as a reference. Thereto, a single viable $S$. aureus cell was immobilized on a tipless cantilever and force-distance curves were recorded in buffer at room temperature. ${ }^{53,54,58}$ From these curves the so-called adhesion force, i.e. the maximum force needed to detach the cell from the surface, was calculated.

Fig. 3 shows the mean adhesion forces of all tested cells on each type of surface. Of note, adhesion forces of $S$. aureus on 

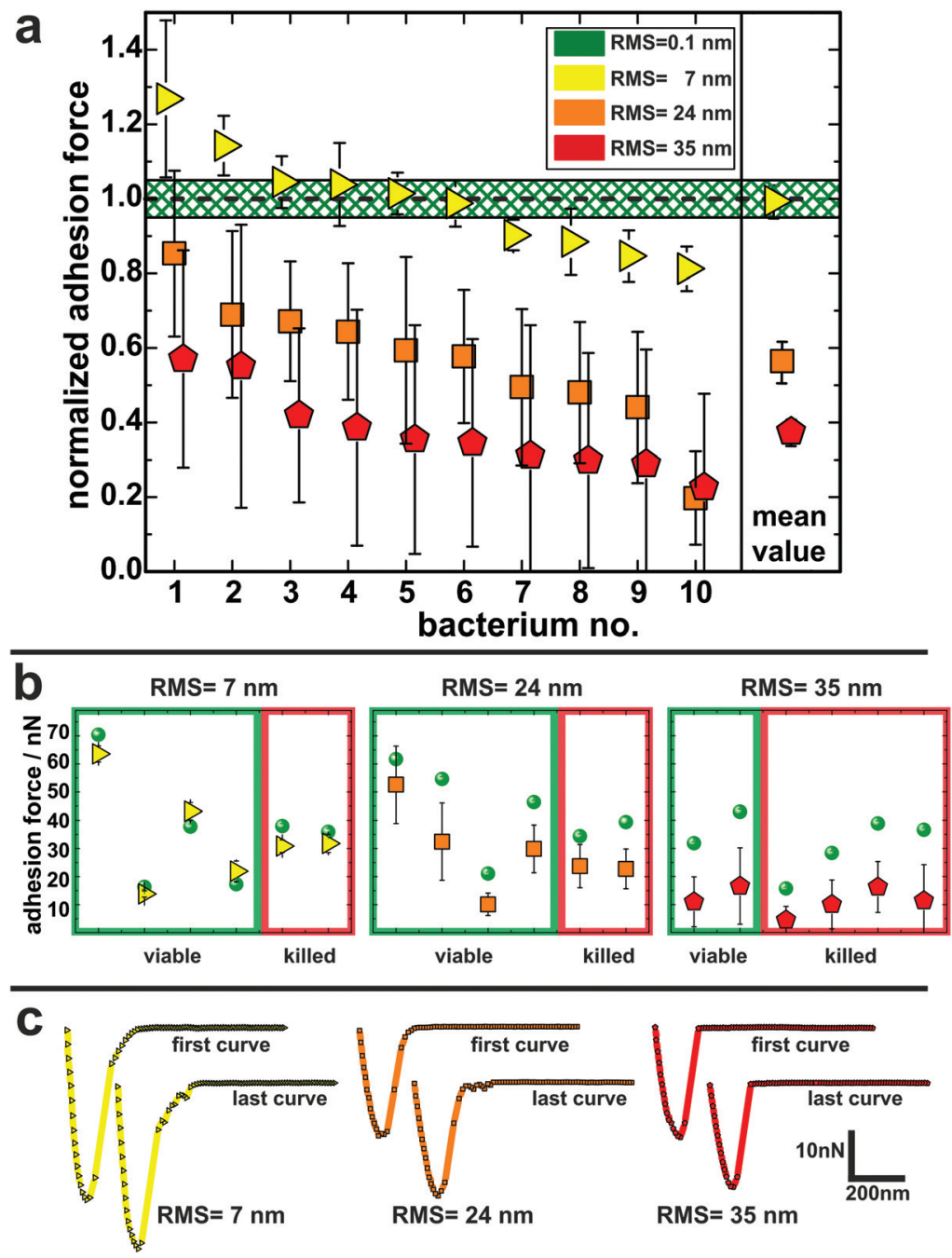

Fig. 3 (a) Adhesion forces of in total 30 S. aureus cells determined on the three types of nanostructured surfaces. Data are normalized to their individual adhesion force on the smooth silicon surface (marked in green). Error bars depict the standard deviation of the adhesion force distribution for every individual cell and - in the case of the mean value - the error of the mean adhesion force determined from every cell (for reasons of clarity, cells are sorted in order of decreasing reduction in adhesion force). (b) Measured adhesion forces of cells that were found to be viable/killed after single-cell force spectroscopy: green spheres show the adhesion force on the smooth surface, colored symbols the adhesion force of the corresponding cell on the nanostructured surfaces. Notably, killing of the cells only occurred after contact to the nanostructured surfaces (if no error bar is shown, it is in fact smaller than the symbol size). (c) First and last force-distance curve recorded on each nanostructured surfaces with an exemplary cell that was found to be killed afterwards.

hydrophobic surfaces are very cell-individual, i.e. they can differ markedly between different cells. ${ }^{50}$ Therefore, for each cell, its mean adhesion force determined on the smooth surface was normalized to 1.0 and its adhesion force on the etched surface was adjusted accordingly (non-normalized values are given in Fig. $3 \mathrm{~b}$ and Fig. S5 in the ESI $\dagger$ ). On the surface with $7 \mathrm{~nm}$ RMS roughness (yellow triangles in Fig. 3a), adhesion forces range from $80-130 \%$ of the forces recorded on the smooth surface with a mean value matching the adhesion force on the smooth surface. On the surface with $24 \mathrm{~nm}$ RMS roughness (orange squares in Fig. 3a), cells feature adhesion forces between $30 \%$ and $90 \%$ of the ones observed on the smooth surface with a mean value of 56(6)\%.§ Adhesion forces on the roughest surface (red pentagons in Fig. 3a) vary between $25 \%$ and $60 \%$ of the forces recorded on the smooth surface. The mean value on the roughest surface is $38(4) \%$ of the value determined on the smooth surface.

The results of the adhesion force measurements can be interpreted in such a way that the contact between a cell and a surface is mediated primarily by cell wall macromolecules tethering to the substrate ${ }^{53,54}$ and thus the adhesive strength of a single cell is determined by the total number of such tethering macromolecules. A recent study indicated that these macromolecules in the $S$. aureus cell wall can extend by approximately $50 \mathrm{~nm}$ due to thermal fluctuations, a length hereinafter referred to as 'tether length'. ${ }^{54}$ The value of $50 \mathrm{~nm}$ for the tether length is confirmed by the snap-in separations obtained from the force-displacement curves of our adhesion 
experiments (for data, see Fig. S7 in the ESI $\dagger$ ). Based on this, Fig. 4 a sketches our proposed molecular picture of bacterial adhesion to nanostructured surfaces: on the smooth silicon surface, a high number or even all surface macromolecules within a certain area, called the contact area, ${ }^{50}$ tether to the surface, and thus adhesion is strongest. Then, with increasing roughness, more and more cell wall macromolecules can no longer reach the surface via thermal fluctuations resulting in a smaller number of tethered molecules. This means that the rougher the surface, the lower the bacterial adhesion force.

In other words, on the nanostructured surfaces, in a certain depth $d$ of the sample (measured from the highest point downwards), only a certain fraction of the total area of the smooth surface is accessible by tethering molecules as visualized in Fig. 4b: for some depth values, e.g. $d_{1}$, the accessible surface area (blue line in Fig. $4 \mathrm{~b}$ ) is smaller than the area of a smooth wafer (green scan line in Fig. $4 \mathrm{~b})$. Then, at a certain depth $\left(d_{2}\right)$ the accessible surface area is approximately equal to the area of a smooth wafer, while for larger depth values, e.g. $d_{3}$, the nanostructured substrate offers more surface area than a smooth wafer. These data are quantitatively shown in Fig. 4c: the accessible surface area of each nanostructured substrate is plotted as a function of the depth from the top of the surface, whereby the data are normalized to the surface area of a smooth wafer as explained in Fig. 4 b.

These curves can be used to explain the adhesion forces on nanostructured substrates in a quantitative way: assuming that the primary reason for reduced adhesion is the reduced accessible surface area, for each curve, this ratio of surface areas equals the ratio of reduced adhesion forces from Fig. 3 (vertical rectangles in Fig. 4c). Thus, the latter can be associated with a corresponding depth that is accessible from the typical top of the surface, whose measurement is described in Fig. S8 in the ESI $\dagger$ (horizontal rectangles in Fig. 4c).

The plot shows that on the surface with $7 \mathrm{~nm}$ RMS roughness, after about $30 \mathrm{~nm}$ from top, the accessible surface area is already at $100 \%$ and all cell wall macromolecules (with an average tether length of $50 \mathrm{~nm}$ ) responsible for adhesion can

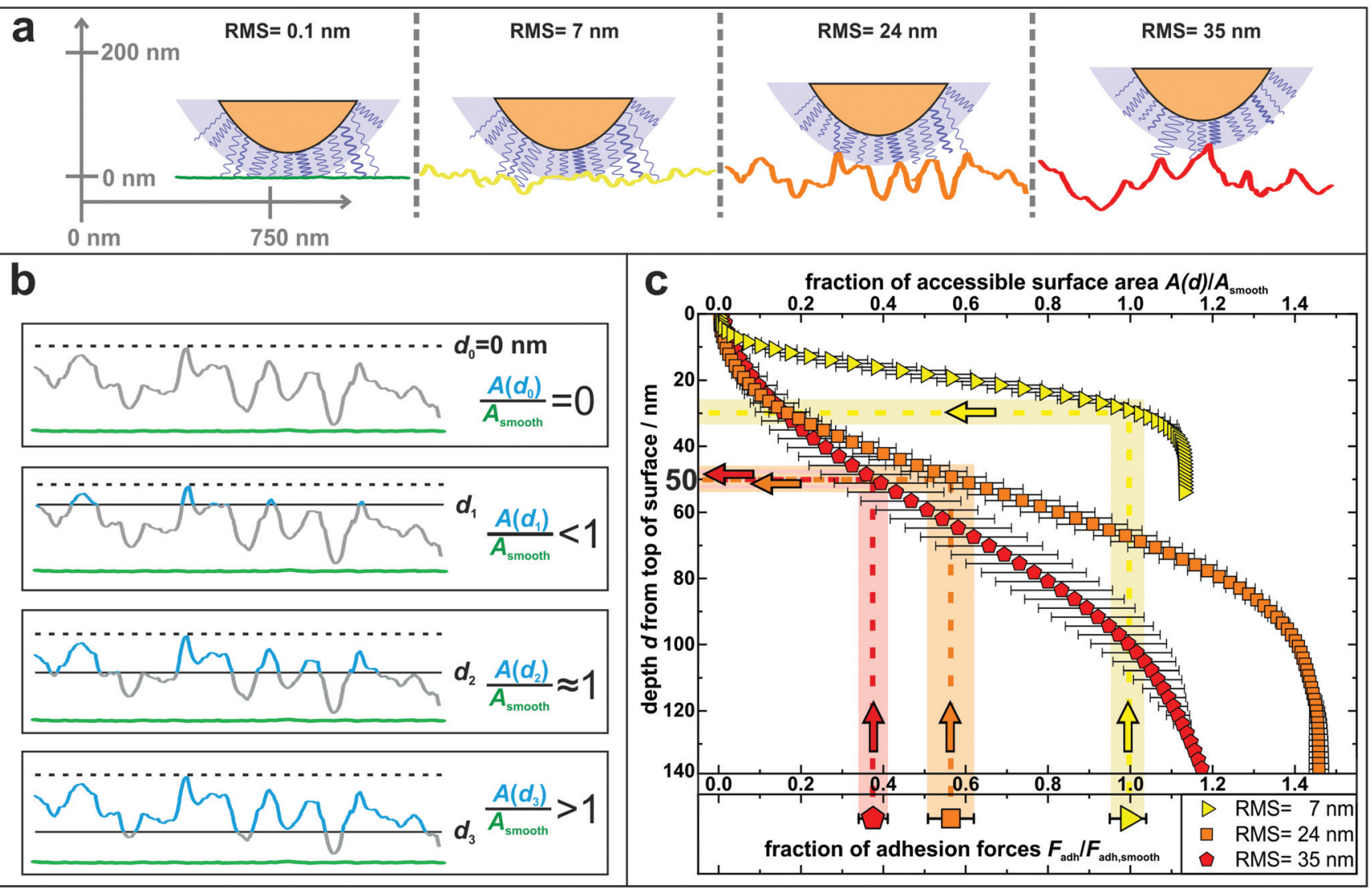

Fig. 4 (a) Sketch to illustrate the adhesion of S. aureus mediated by macromolecular tethering on different nanostructured surfaces: the bigger the surface structures, the lower is the number of macromolecules that are able to reach the substrate. Notably, only few molecules are drawn on the cell wall, whereas their density is in reality much higher, as symbolized by the blue shaded layer. All surfaces are represented by real AFM scan lines. (b) Visual explanation of the data shown in part c: accessible surface area (blue lines) of a nanostructured substrate compared to the surface area of a smooth substrate (green lines) for four exemplary depth values $d_{0}-d_{3}$. (c) Depth $d$ from top of the surface and corresponding fraction of accessible surface area of all nanostructured substrates compared to the total surface area of a smooth substrate. The light-colored vertical rectangles show the relative decrease of adhesion forces on the nanostructured surfaces (the center line of each rectangle indicates the mean value of adhesion forces and the width corresponds to the error of the mean from Fig. 3). The horizontal rectangles indicate the corresponding depth in which this fraction of surface area is accessible. 
reach the surface and, therefore, the adhesive strength is the same as on the smooth wafer. Some cells even show a stronger adhesion than on the smooth surface, which can be explained, besides by statistical fluctuations, by the fact that the total surface area on the nanostructured substrate is - for distances from the top greater than $30 \mathrm{~nm}$ - larger than on the smooth wafer. Hence, cell wall macromolecules with tether lengths of $50 \mathrm{~nm}$ can 'find' even more binding sites resulting in stronger adhesion. ${ }^{59,60}$ For the surfaces with $24 \mathrm{~nm}$ and $35 \mathrm{~nm}$ RMS roughness, the ratios of surface areas that correspond to the reduced adhesion forces of 56(6)\% and 38(4)\% are both accessible in a depth of $45-55 \mathrm{~nm}$. This depth perfectly matches the $S$. aureus tether length of $50 \mathrm{~nm}$, a fact that strongly supports our hypothesis that the reason for reduced adhesion forces is primarily caused by the reduced accessible surface area.

The quantitative influence of surface roughness on the adhesion of $S$. aureus cells can probably be transferred to other (Gram-positive) bacteria (see Fig. S9 in the ESI $\uparrow$ for experiments with Staphylococcus carnosus). However, it should be mentioned that the exact range of surface roughness that best prevents adhesion depends on the average tether length of the cell wall macromolecules and in part on the contact area. Both quantities may vary for different species. In addition, care should be taken when transferring our results to surfaces where other effects, such as surface deformations due to rather flexible structures (see Fig. S10 in the ESI $†$ ), may dominate the adhesive behaviour of bacteria. The same might be true for the bactericidal properties of our substrates which are described below.

Since other studies have reported bactericidal effects of surfaces with structures similar to those of our substrates, ${ }^{61-64}$ it was of particular interest for us to determine the impact of the nano-topography on the viability of the cells used in our study. Therefore, we performed live/dead staining for some of the cells after adhesion measurements. In doing so, we also checked if there is a correlation between the measured adhesion of particular cells and their viability after the experiment. It shows that the fraction of dead cells increases with increasing size of surface structures: on the smooth silicon, none of the tested cells were killed. On the surfaces with $7 \mathrm{~nm}$ and $24 \mathrm{~nm}$ RMS roughness, one third of the tested cells were killed and on the roughest surface, two thirds of the tested cells were found dead after measurements. Interestingly, no correlation between the adhesion force (or the change in adhesion force) and the viability of the corresponding cell can be observed in adhesion force measurements (Fig. 3b). Accordingly, the characteristic shape of the force-distance curves did not change in the course of several measurements with cells that were found to be dead at the end of the experiment (Fig. 3c). Hence, it seems that, surprisingly, the viability of a cell does not influence its adhesive strength - at least not for the time scales (approximately one hour) and the conditions of our experiments and for the used surfaces.

In the literature, the bactericidal properties of structured surfaces are usually attributed to the penetration or intrusion and subsequent stretching of the bacterial cell wall by 'spiky' features on the substrate. ${ }^{61-64}$ On the one hand, our experiments suggest that the size of these features on the roughest surface is generally most effective for killing because the fraction of dead cells is the largest on this substrates. On the other hand, cell death on a certain surface seems to depend on the particular cell respectively its locally varying cell wall properties at the contact area.$^{65}$ For some cells, the layer of cell wall molecules may be (locally) very dense and/or thick and therefore so 'strong' that it prevents the spiky structures from deeply intruding the cell wall. Other cells, in contrast, may have a less dense and/or thinner rather 'weak' macromolecular layer and can, therefore, be penetrated by the spiky structures. Interestingly, the adhesion force of an individual cell is not correlated to its viability after adhesion force measurement (see Fig. 3b). Therefore, we can conclude that a more 'robust' macromolecular layer is not necessarily a more 'adhesive' layer. Notably, we determined the viability only for a limited number of cells. Hence, to give a general statement about the bactericidal effect of our nanostructured surfaces, the number of these measurements should be increased and additional experiments (for example, in a flow chamber setup) should be performed.

\section{Conclusions}

In summary, we have shown that surface morphometry is quantitatively related to the strength of bacterial adhesion on surfaces with a nano-scaled topography. Our single-cell force spectroscopy measurements on etched silicon surfaces revealed that the adhesion force of $S$. aureus cells to nanostructured substrates decreases with increasing size of surface structures. The most important outcome is that a detailed characterization by Minkowski functionals allows a direct correlation between surface nanostructures and bacterial adhesion forces: since the adhesive strength is determined by the number of cell wall macromolecules tethering to the surface, the reduced accessible surface area on the nanostructured substrates for cell wall molecules with average tether lengths of $50 \mathrm{~nm}$ matches the reduced mean adhesion forces on the nano-topographies remarkably well. Quantifying the variances of tether lengths, surface areas and adhesion forces and determining whether they are also related to each other is an interesting question for future research.

In addition, we have shown that the nanostructure of our substrates influences the viability of bacteria after contact: similar to the adhesive strength, the percentage of viable cells on the nanostructured surfaces decreases with increasing surface roughness while the adhesive strength of individual cells is not influenced by their viability.

Our results can be of importance in industry and medicine since nanostructured surfaces are increasingly used in various applications (for example, as materials for bioreactors or prosthetics). ${ }^{2,3}$ For scenarios, in which bacterial adhesion is unwanted, our results can provide suggestions regarding surface topography: while molecularly smooth surfaces or 
those with structures larger than the cells display a favorable adhesion ground for bacteria, this property is markedly reduced on surfaces that feature structures in the same size range as the macromolecules of the bacterial cell wall. At the same time, this size range also seems to be effective for killing cells by contact with these structures. Furthermore, our study shows the strong potential of surface characterization using Minkowksi functionals since this method provides universal morphological information allowing, for example, a precise comparison of different surfaces.

\section{Materials and methods}

\section{Silicon wafers}

Silicon wafers in (100) orientation with a resistivity of 10-20 $\Omega \mathrm{cm}$ were purchased from Siltronic (Burghausen, Germany). They feature a native silicon oxide layer with a thickness of $1.7(2) \mathrm{nm}$ as determined by ellipsometry. ${ }^{66}$ The wafers are by default polished to an RMS roughness of $0.09(2) \mathrm{nm}$ as determined by AFM. ${ }^{66}$

\section{Preparation of the nanostructured surfaces}

In a first step, silicon surfaces were covered with a gold layer of a nominal film thickness of $2 \mathrm{~nm}$ which was determined with a quartz crystal microbalance by physical vapor deposition. As is known, this procedure does not result in a continuous gold film, but in gold clusters of sub-micron size. ${ }^{52}$ Subsequently, following a recipe by Koynov et al., ${ }^{51}$ the gold-covered silicon was etched by immersing the wafers in a mixture of fluoric acid ( $\mathrm{HF}, 40 \%)$, hydrogen peroxide $\left(\mathrm{H}_{2} \mathrm{O}_{2}, 35 \%\right)$ and water for $90 \mathrm{~s}, 180 \mathrm{~s}$ and $360 \mathrm{~s}$, respectively. Residues from the etching solution were removed by extensively rinsing the etched wafers in ultra-pure water. After etching, the gold clusters were removed by immersing the wafers in aqua regia (1:3 mixture of $\mathrm{HNO}_{3}(65 \%)$ and $\left.\mathrm{HCl}(37 \%)\right)$ for $25 \mathrm{~min}$. Afterwards, the wafers were rinsed again with ultra-pure water to remove possible leftovers of the acid. X-ray photoelectron spectroscopy (XPS) confirmed that no gold was present on the surface after this treatment (an XPS overview spectrum is given in Fig. S1a $\dagger$ ). To render the wafers hydrophobic, they were covered with a self-assembling monolayer of silanes according to a standard recipe. ${ }^{55}$ Right before every set of experiments with bacteria, all surfaces were cleaned in ultrasonic baths of ethanol and acetone for $3 \mathrm{~min}$ each. After cleaning, the surfaces were dried under a stream of pure nitrogen.

\section{Bacteria}

For this study, cells of the S. aureus strain SA113 were used. Bacteria from a deep-frozen stock solution were grown on a blood agar plate for 2.5 days at $37^{\circ} \mathrm{C}$. Such a plate was used for two weeks at the maximum. For the experiments, one colony from the plate was transferred into $5 \mathrm{ml}$ of sterile tryptic soy broth (TSB) and cultured for $16 \mathrm{~h}$ at $37^{\circ} \mathrm{C}$ under agitation $(150 \mathrm{rpm})$. From this culture, $40 \mu \mathrm{l}$ were transferred into $4 \mathrm{ml}$ of fresh TSB and cultured once more for $2.5 \mathrm{~h}$ at $37^{\circ} \mathrm{C}$ and $150 \mathrm{rpm}$. Finally, $1 \mathrm{ml}$ was taken from this culture and washed three times by centrifuging for $3 \mathrm{~min}$ at $17000 \mathrm{~g}$, replacing the supernatant by $1 \mathrm{ml}$ of fresh phosphate buffered saline (PBS, pH 7.3) and thoroughly vortexing. This procedure results in a bacterial suspension with an optical density at $600 \mathrm{~nm}\left(\mathrm{OD}_{600}\right)$ of $0.2-0.3$.

\section{Viability measurements}

To check the viability of the cells, the BacLight assay purchased from Molecular Probes, Eugene, USA was used. It consists of Syto 9 and propidium iodide. After excitation, the Syto 9 stain emits green light when bound to nucleic acids in bacteria. In contrast, propidium iodide, which can only enter the porous cell wall of dead bacterial cells, emits red light and causes a reduction of the binding between the Syto 9 stain and the bacterial nucleic acids. Hence, after staining a bacterial solution with the BacLight mixture and illuminating it with white light, viable cells glow green and dead cells red. After adhesion measurements, we placed the used cantilever including the immobilized cell in a drop $(1 \mathrm{ml})$ of the staining assay. To achieve best results, the stain was left to act on the cell for $10 \mathrm{~min}$ in the dark. Thereafter, its residues were removed by carefully replacing the drop by fresh PBS for three times. Care was taken that the cantilever with the cell never was completely dry. Then, the cell on the cantilever was observed by fluorescence microscopy.

\section{Force-distance measurements}

We recorded force-distance curves on a Bioscope Catalyst (Bruker-Nano, Santa Barbara, USA) with single viable bacterial cells immobilized on a tipless cantilever (MLCT-0, BrukerNano) with nominal spring constants of $0.01 \mathrm{~N} \mathrm{~m}^{-1}$ and $0.3 \mathrm{~N} \mathrm{~m}^{-1}$. A detailed description of how the cells were attached to the cantilevers can be found in the paper by Thewes et al. ${ }^{67}$ in short: tipless cantilevers were cleaned in an air-plasma and afterwards covered with a thin layer of dopamine by immersing them in a solution of $4 \mathrm{mg} \mathrm{ml}^{-1}$ dopamine hydrochloride in TRIS/HCL-buffer for one hour. The cantilevers were then cleaned in ultrapure water and dried in a clean room environment. The cantilevers' spring constant and deflection sensitivity were calibrated before each set of measurements. To pick up a single cell, a very dilute bacterial suspension was placed on a Petri dish that was observed with an inverse optical microscope equipped with a micromanipulator. The calibrated cantilever is then connected to the micromanipulator and approached from above to the sedimented bacterial cells in the Petri dish. In a final step, the cantilever is carefully lowered onto a single cell and gently pressed onto it so that the cell adheres to the cantilever. This bacterial probe is afterwards carefully inserted in the microscope without drying out the cell.

For every force-distance curve, the approach and retraction distance was $800 \mathrm{~nm}$ with a retraction velocity of $800 \mathrm{~nm} \mathrm{~s}^{-1}$. The force trigger, i.e. the force with which the cell is pressed onto the surface, was set to $300 \mathrm{pN}$ which does hardly deform the cell or change its contact area ${ }^{50,68}$ (for a more detailed dis- 
cussion of the chosen force trigger, see the ESI†). Curves were recorded with $0 \mathrm{~s}$ surface delay time which corresponds to a 'real' contact time below $0.5 \mathrm{~s}^{53,58}$ For each individual cell, 100 force-distance curves were recorded on the smooth as well as on a nanostructured surface in a rectangular pattern with a distance of $1 \mu \mathrm{m}$ between each curve. To exclude that probing the smooth/nanostructured surfaces might alter the adhesive behaviour of the bacterial cell, the first 50 curves were recorded on the smooth surface and the next 50 were recorded on the nanostructured surface. Then, again, the smooth surface and afterwards the nanostructured surface was probed by 50 curves each. By comparing the adhesion of each set of curves on the smooth/nanostructured surface, it was ensured that the adhesion behavior was not changed during the recording of several force-distance curves on the different surfaces (as it was also seen before on smooth silicon ${ }^{53,68}$ ).

\section{AFM surface topography measurements}

The surface topography was measured in tapping mode with an Icon FastscanBio (Bruker-Nano, Santa Barbara, USA) in air using high aspect ratio tips (HAR1-200-10, Bruker-Nano) with a nominal spring constant of $42 \mathrm{~N} \mathrm{~m}^{-1}$ in soft tapping mode with an image resolution of $1024 \times 1024$ pixels. The scan area was $1 \mu \mathrm{m} \times 1 \mu \mathrm{m}$ for the $90 \mathrm{~s}$ and $180 \mathrm{~s}$ etched substrates. The $360 \mathrm{~s}$ etched surface fluctuates strongly and so do its geometrical properties. To reduce the statistical error, we scanned a larger observation window of $3 \mu \mathrm{m} \times 3 \mu \mathrm{m}$. Systematic effects of the lower physical resolution on the total surface area should be less than $3 \%$, as estimated from the other surface when we artificially reduced the resolution. The resolution in $z$-direction was below $0.5 \mathrm{~nm}$. From these images, the AFM tip shape was determined and the real surface was reconstructed by deconvoluting the recorded image and the tip geometry using the software Gwyddion. For every type of surface, several AFM images were recorded and analyzed, providing average values and standard deviations for RMS roughnesses and Minkowski functionals.

\section{Minkowski analysis}

The level sets of the AFM images and their three-dimensional triangulations were analyzed using Minkowski functionals. In the latter case, the triangulation was constructed using the 'Advancing Front Surface Reconstruction' from CGAL. ${ }^{56}$ Using the 3D Minkowski software Karambola, ${ }^{47}$ the surface area was computed as a function of the height. More precisely, as a function of the difference in height to the maximal peak within the observation window, the surface area was computed for all those triangles whose lowest vertex was above this threshold. In the first case, the AFM images were converted into pixelated gray scale maps and then into black-and-white images via thresholding. A pixel whose height is above a threshold $h$ turns white, all others become black. The three two-dimensional Minkowski functionals of the white domains were then computed as a function of the threshold height $h$ using the software Papaya. ${ }^{46}$ To reduce the pixelization errors, a standard marching square algorithm was applied. ${ }^{45}$ Edge effects from the observation window were avoided by using minus-sampling boundary conditions (the outermost pixels were used for the boundary conditions). To avoid dependencies on lateral dimensions of the AFM scans so-called densities of Minkowski functionals are used, i.e. the functionals are rescaled by the size of the observation window.

\section{Electronmicroscopy measurements}

For the scanning electron microscopy (SEM) measurements, a FEI (Hilsboro, USA) Quanta 400 FEG SEM in high vacuum mode was used. Secondary electron images were collected at $10 \mathrm{kV}$ and $15 \mathrm{kV}$ accelerating voltage under different tilting angles.

\section{Author contributions}

C.S., N.T., M.B., K.J. designed research. C.S., F.N., J.M. performed AFM and viability measurements. S.G., F.M. performed XPS measurements. M.K. performed REM measurements. M.A.K. performed Minkowski analyses. C.S., F.N., J.M., T.F., S.G., F.M., M.A.K. analyzed data. C.S., M.B., M.A.K., K.J. wrote the manuscript with the help of all other authors.

\section{Conflicts of interest}

There are no conflicts to declare.

\section{Acknowledgements}

The authors thank Günter Marchand (Mitranz Mikrotechnologie-Transferzentrum, Saarland University) for etching the substrates. Work was funded by the German Research Foundation (DFG) within the framework of the Collaborative Research Center SFB 1027 (project B2). M. A. K. acknowledges support of the DFG through the research group 'Geometry and Physics of Spatial Random Systems' (GPSRS) under grants number HU1874/3-2, and LA965/6-2.

\section{Notes and references}

$\S$ Values in brackets indicate the standard error of the mean.

1 J. W. Costerton and L. Montanaro, Int. J. Artif. Organs, 2007, 30, 757-763.

2 D. Neut, H. C. van der Mei, S. K. Bulstra and H. J. Busscher, Acta Orthop., 2007, 78, 299-308.

3 J. A. Callow and M. E. Callow, Nat. Commun., 2011, 2, 244.

4 O. Lieleg, M. Caldara, R. Baumgärtel and K. Ribbeck, Soft Matter, 2011, 7, 3307-3314.

5 C. S. Laspidou, L. A. Spyrou and N. Aravas, Math. Biosci., 2014, 251, 11-15.

6 K. Anselme, P. Davidson, A. M. Popa, M. Giazzon, M. Liley and L. Ploux, Acta Biomater., 2010, 6, 3824-3846. 
7 L. Rizzello, B. Sorce, S. Sabella, G. Vecchio, A. Galeone, V. Brunetti, R. Cingolani and P. P. Pompa, ACS Nano, 2011, 5, 1865-1876.

8 Y. Lu, S. Sathasivam, J. Song, C. R. Crick, C. J. Carmalt and I. P. Parkin, Science, 2015, 347, 1132-1135.

9 D. P. Linklater, M. De Volder, V. A. Baulin, M. Werner, S. Jessl, M. Golozar, L. Maggini, S. Rubanov, E. Hanssen, S. Juodkazis and E. P. Ivanova, ACS Nano, 2018, 12, 66576667.

10 J. Li, K. Zhang, L. Ruan, S. F. Chin, N. Wickramasinghe, H. Liu, V. Ravikumar, J. Ren, H. Duan, L. Yang, et al., Nano Lett., 2018, 18, 4180-4187.

11 E. P. Ivanova, V. K. Truong, J. Y. Wang and C. C. Berndt, Langmuir, 2010, 26, 1973-1982.

12 V. K. Truong, S. Rundell, R. Lapovok and Y. Estrin, Appl. Microbiol. Biotechnol., 2009, 83, 925-937.

13 S. H. Flint, J. D. Brooks and P. J. Bremer, J. Food Eng., 2000, 43, 235-242.

14 L. C. Hsu, J. Fang and D. A. Borca, Appl. Environ. Microbiol., 2013, 79, 2703.

15 S. D. Puckett, E. Taylor, T. Raimondo and T. J. Webster, Biomaterials, 2010, 31, 706-713.

16 S. Kang and M. Elimelech, Langmuir, 2009, 25, 96569659.

17 S. El-Kirat-Chatel, A. Beaussart, C. D. Boyd, G. A. O'Toole and Y. F. Dufrêne, ACS Chem. Biol., 2013, 9, 485-494.

18 A. Beaussart, S. El-Kirat-Chatel, P. Herman, D. Alsteens, J. Mahillon, P. Hols and Y. F. Dufrêne, Biophys. J., 2013, 104, 1886-1892.

19 A. Beaussart, S. El-Kirat-Chatel, R. M. A. Sullan, D. Alsteens, P. Herman, S. Derclaye and Y. F. Dufrêne, Nat. Protoc., 2014, 9, 1049.

20 A. Beaussart, M. Abellán-Flos, S. El-Kirat-Chatel, S. P. Vincent and Y. F. Dufrêne, Nano Lett., 2016, 16, 12991307.

21 C. Formosa-Dague, C. Feuillie, A. Beaussart, S. Derclaye, S. Kucharíková, I. Lasa, P. Van Dijck and Y. F. Dufrêne, ACS Nano, 2016, 10, 3443-3452.

22 C. Formosa-Dague, Z.-H. Fu, C. Feuillie, S. Derclaye, T. J. Foster, J. A. Geoghegan and Y. F. Dufrêne, Nanoscale Horiz., 2016, 1, 298-303.

23 C. Spengler, N. Thewes, F. Nolle, T. Faidt, N. Umanskaya, M. Hannig, M. Bischoff and K. Jacobs, J. Mol. Recognit., 2017, 30, e2615.

24 Y. F. Dufrêne, ACS Nano, 2017, 11, 19-22.

25 T. D. Becke, S. Ness, R. Gürster, A. F. Schilling, A.-M. di Guilmi, S. Sudhop, M. Hilleringmann and H. ClausenSchaumann, ACS Nano, 2018, 12, 549-558.

26 C. Feuillie, C. Valotteau, L. Makart, A. Gillis, J. Mahillon and Y. F. Dufrêne, Nano Lett., 2018, 18, 5821-5826.

27 T. R. Scheuerman, A. K. Camper and M. A. Hamilton, J. Colloid Interface Sci., 1998, 208, 23-33.

28 A. I. Hochbaum and J. Aizenberg, Nano Lett., 2010, 10, 3717-3721.

29 C. Díaz, P. L. Schilardi and R. C. Salvarezza, Langmuir, 2007, 23, 11206-11210.
30 D. Perera-Costa, J. M. Bruque, M. L. González-Martín, A. C. Gómez-García and V. Vadillo-Rodríguez, Langmuir, 2014, 30, 4633-4641.

31 N. Lu, W. Zhang, Y. Weng, X. Chen, Y. Cheng and P. Zhou, Food Control, 2016, 68, 344-351.

32 K. K. Sakimoto, C. Liu, J. Lim and P. Yang, Nano Lett., 2014, 14, 5471-5476.

33 Y. Wu, J. P. Zitelli, K. S. TenHuisen, X. Yu and M. R. Libera, Biomaterials, 2011, 32, 951-960.

34 V. K. Truong, V. Pham and A. Medvedev, Appl. Microbiol. Biotechnol., 2015, 99, 6831-6840.

35 E. P. Ivanova, V. K. Truong, H. K. Webb and V. A. Baulin, Sci. Rep., 2011, 1, 165.

36 A. V. Singh, V. Vyas, R. Patil, V. Sharma and P. E. Scopelliti, PLoS One, 2011, 6, e25029.

37 E. Gadelmawla, M. Koura, T. Maksoud, I. Elewa and H. Soliman, J. Mater. Process. Technol., 2002, 123, 133-145.

38 R. J. Crawford, H. K. Webb, V. K. Truong, J. Hasan and E. P. Ivanova, Adv. Colloid Interface Sci., 2012, 179, 142-149.

39 G. E. Schröder-Turk, W. Mickel, S. C. Kapfer, M. A. Klatt, F. M. Schaller, M. J. F. Hoffmann, N. Kleppmann, P. Armstrong, A. Inayat, D. Hug, M. Reichelsdorfer, W. Peukert, W. Schwieger and K. Mecke, Adv. Mater., 2011, 23, 2535-2553.

40 R. Schneider and W. Weil, Stochastic and Integral Geometry (Probability and Its Applications), Springer, Berlin, 2008.

$41 \mathrm{H}$. Hadwiger, Vorlesungen über Inhalt, Oberfläche und Isoperimetrie, Springer, Berlin, 1957.

42 M. A. Klatt, G. E. Schröder-Turk and K. Mecke, Med. Phys., 2017, 44, 3663-3675.

43 K. Mecke, Int. J. Mod. Phys. B, 1998, 12, 861-899.

44 K. Jacobs, R. Seemann and K. Mecke, Statistical Physics and Spatial Statistics, Springer, 2000, 72-91.

45 H. Mantz, K. Jacobs and K. Mecke, J. Stat. Mech.: Theory Exp., 2008, 12, P12015.

46 G. E. Schröder-Turk, S. Kapfer, B. Breidenbach, C. Beisbart and K. Mecke, J. Microsc., 2010, 238, 57-74.

47 G. E. Schröder-Turk, W. Mickel, S. C. Kapfer, F. M. Schaller, B. Breidenbach, D. Hug and K. Mecke, New J. Phys., 2013, 15, 083028.

48 D. Göring, M. A. Klatt, C. Stegmann and K. Mecke, Astron. Astrophys., 2013, 555, A38.

49 F. D. Lowy, N. Engl. J. Med., 1998, 339, 520-532.

50 C. Spengler, N. Thewes, P. Jung, M. Bischoff and K. Jacobs, Nanoscale, 2017, 9, 10084-10093.

51 S. Koynov, M. S. Brandt and M. Stutzmann, Appl. Phys. Lett., 2006, 88, 203107.

52 I. Doron-Mor, Z. Barkay, N. Filip-Granit, A. Vaskevich and I. Rubinstein, Chem. Mater., 2004, 16, 3476-3483.

53 N. Thewes, P. Loskill, P. Jung, H. Peisker, M. Bischoff, M. Herrmann and K. Jacobs, Beilstein J. Nanotechnol., 2014, 5, 1501-1512.

54 N. Thewes, A. Thewes, P. Loskill, H. Peisker, M. Bischoff, M. Herrmann, L. Santen and K. Jacobs, Soft Matter, 2015, 11, 8913-8919. 
55 M. Lessel, O. Bäumchen, M. Klos, H. Hähl, R. Fetzer, M. Paulus, R. Seemann and K. Jacobs, Surf. Interface Anal., 2015, 47, 557-564.

56 T. K. F. Da and D. Cohen-Steiner, CGAL User and Reference Manual, CGAL Editorial Board, 4.12 edn, 2018.

57 P. Loskill, H. Hähl, N. Thewes, C. T. Kreis, M. Bischoff, M. Herrmann and K. Jacobs, Langmuir, 2012, 28, 72427248.

58 A. Beaussart, P. Herman and S. El, Nanoscale, 2013, 5, 10894-10900.

59 R. D. Boyd, J. Verran, M. V. Jones and M. Bhakoo, Langmuir, 2002, 18, 2343-2346.

60 K. A. Whitehead, D. Rogers, J. Colligon, C. Wright and J. Verran, Colloids Surf., B, 2006, 51, 44-53.

61 E. P. Ivanova, J. Hasan, H. K. Webb and V. K. Truong, Small, 2012, 8, 2489-2494.

62 E. P. Ivanova, J. Hasan, H. K. Webb, G. Gervinskas, S. Juodkazis, V. K. Truong, A. H. Wu, R. N. Lamb,
V. A. Baulin, G. S. Watson, J. A. Watson, D. E. Mainwaring and R. J. Crawford, Nat. Commun., 2013, 4, 2838.

63 S. Pogodin, J. Hasan, V. A. Baulin, H. K. Webb and V. K. Truong, Biophys. J., 2013, 104, 835-840.

64 C. M. Bhadra, V. K. Truong, V. Pham and M. Al Kobaisi, Sci. Rep., 2015, 5, 16817.

65 J. M. Monteiro, P. B. Fernandes, F. Vaz, A. R. Pereira, A. C. Tavares, M. T. Ferreira, P. M. Pereira, H. Veiga, E. Kuru, M. S. VanNieuwenhze, et al., Nat. Commun., 2015, 6, 8055 .

66 M. Bellion, L. Santen, H. Mantz, H. Hähl, A. Quinn, A. Nagel, C. Gilow, C. Weitenberg and Y. Schmitt, J. Phys.: Condens. Matter, 2008, 20, 404226.

67 N. Thewes, P. Loskill, C. Spengler, S. Hümbert, M. Bischoff and K. Jacobs, Eur. Phys. J. E: Soft Matter Biol. Phys., 2015, 38, 140 .

68 P. Loskill, P. M. Pereira, P. Jung, M. Bischoff, M. Herrmann, M. G. Pinho and K. Jacobs, Biophys. J., 2014, 107, 1082-1089. 